\title{
ACCURACY ASSESSMENT OF A CANAL-TUNNEL 3D MODEL BY COMPARING PHOTOGRAMMETRY AND LASERSCANNING RECORDING TECHNIQUES
}

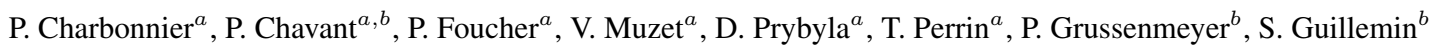 \\ ${ }^{a}$ CETE de l'Est, LRPC de Strasbourg, 11 rue Jean Mentelin, 67035 Strasbourg, France \\ \{Pierre.Charbonnier, Philippe.Foucher, Valerie.Muzet, Davy.Prybyla, Thibaut.Perrin\}@ developpement-durable.gouv.fr \\ ${ }^{b}$ ICube Laboratory UMR 7357, Photogrammetry and Geomatics Group, \\ INSA de Strasbourg, 24 Bd de la Victoire, 67084 Strasbourg, France \\ \{paul.chavant, pierre.grussenmeyer,samuel.guillemin,\}@insa-strasbourg.fr
}

KEY WORDS: Photogrammetry, Cultural Heritage, Laser Scanning, Point Cloud, Model, Recording, Accuracy, Comparison

\begin{abstract}
:
With recent developments in the field of technology and computer science, conventional methods are being supplanted by laser scanning and digital photogrammetry. These two different surveying techniques generate 3-D models of real world objects or structures. In this paper, we consider the application of terrestrial Laser scanning (TLS) and photogrammetry to the surveying of canal tunnels. The inspection of such structures requires time, safe access, specific processing and professional operators. Therefore, a French partnership proposes to develop a dedicated equipment based on image processing for visual inspection of canal tunnels. A 3D model of the vault and side walls of the tunnel is constructed from images recorded onboard a boat moving inside the tunnel. To assess the accuracy of this photogrammetric model (PM), a reference model is build using static TLS. We here address the problem comparing the resulting point clouds. Difficulties arise because of the highly differentiated acquisition processes, which result in very different point densities. We propose a new tool, designed to compare differences between pairs of point cloud or surfaces (triangulated meshes). Moreover, dealing with huge datasets requires the implementation of appropriate structures and algorithms. Several techniques are presented : point-to-point, cloud-to-cloud and cloud-to-mesh. In addition farthest point resampling, octree structure and Hausdorff distance are adopted and described. Experimental results are shown for a $475 \mathrm{~m}$ long canal tunnel located in France.
\end{abstract}

\section{INTRODUCTION}

In France, 31 tunnels were built during the 19th and 20th century, totalling $42 \mathrm{~km}$ of underground waterway. Inspecting canal tunnels is not only a matter of heritage preservation, but also a safety issue. However, on-site visual inspections are time-consuming and may interfere with navigation traffic. In this context, a French consortium, composed of Voies Navigables de France (the French operator of waterways), the Centre d'Etudes des Tunnels (CETU) and the CETE de l'Est, in collaboration with the Photogrammetry and Geomatics Group (INSA), proposes to develop a visual inspection system, based on image recording, for helping the operator's task. In this way, a prototype has been developed to dynamically record images of the vault and side walls of the canal tunnel and a 3D photogrammetric model of the tunnel was built.

The main objective of this paper is to assess the accuracy of the photogrammetric model by taking as reference a static terrestrial laser scanning (TLS) model. A technique to evaluate accuracy is to compute a distance between a dataset and a $3 \mathrm{D}$ reference point cloud. However, calculating such a distance may be problematic. In particular, the two distinct acquisition processes lead to different number of points in both models. Moreover, the point density within the two clouds is non-uniform. Note that laser scanning stations are located on a bench, near a side wall, which implies an heterogeneous density of the cloud between both sides of the tunnel. Finally, the tunnel is quite long and the mass of recorded data may be very huge. Therefore specific computation structures will be required.

We are interested in three different algorithms that compute a distance between the two models : point-to-point, cloud-to-mesh and cloud-to-cloud methods. Facing huge datasets, we propose to implement these algorithms using a specific octree structure, for its capacity to quickly and accurately address points at a given location. Differences in sampling may be alleviated either by using meshing or by considering non-symmetric distances. The second contribution of the paper is that we evaluate the accuracy of the photogrammetric model. First, we need to check the reliability of the reference TLS model. Subsequently, we compare the photogrammetric model to the TLS model by using the three proposed accuracy measures.

The paper is organized as follows. We first propose an overview of related works (Sec. 2). We introduce the three approaches we propose for comparing huge datasets (Sec. 3). In Sec. 4, we present the experimental setup for data recording. In Sec. 5, we comment the experimental results. Finally, Sec. 6 is dedicated to conclusions and future work.

\section{RELATED WORK}

Photogrammetry and lasergrammetry have become relevant to inspect tunnels. These new technologies have been introduced to measure displacements (Kim et al., 2007) or monitor deformations on tunnel profiles (Han and Jiang, 2013). In (Pejić, 2013), an optimized laserscanning solution is proposed for inspecting the geometry of railway tunnels.

Some tools were reported, that estimate Point-to-mesh and meshto-mesh distances between two triangulated meshes (Cignoni et al., 1998, Aspert et al., 2002) on small point files. In (Cignoni et al., 1998), it may be noticed that the distance is computed between an original mesh and its simplified representation. In (Mémoli and Sapiro, 2004), the authors introduce an approach based on the Hausdorff distance, for comparing point clouds. However, this formal approach requires an uniform sampling. Our study deals with huge datasets, which implies using either 
an appropriated sampling (Eldar et al., 1997, Bronstein et al., 2008) or specific computation structures such as an octree subdivision (Girardeau-Montaut et al., 2005). An original method is proposed in (Lague et al., 2013) for surveying canyon surface changes using TLS. In this approach, the distance between two point clouds (Laser clouds and dense tacheometric survey) is obtained by locally estimating the surface normals and computing the distance in the direction of the normals. A roughness measure is considered for computing the surface normals and a local confidence range is calculated.

\section{METHODOLOGY}

We propose three tools to evaluate the accuracy of the photogrammetric model. In the first method, point-to-point distances are computed. The second approach computes Euclidean distances between photogrammetric points and lasergrametric meshes. In the third approach, the Hausdorff distance $\left(d_{H}\right)$ is used to compare two point clouds ( $S 1$ and $S 2$ ). In this section, we first recall the basis of Hausdorff distance and of the octree structure. the we introduce the three proposed approaches to measure an accuracy.

\subsection{Hausdorff distance}

The Hausdorff distance $d_{H}$ is a classical tool that measures the remoteness between two subsets $S_{1}$ and $S_{2}$ of a metric space:

$$
d_{H}(S 1, S 2)=\max [d(S 1, S 2), d(S 2, S 1)],
$$

where $d(S 1, S 2)$ and $d(S 2, S 1)$ are symmetrically defined as:

$$
\begin{aligned}
& d(S 1, S 2)=\max _{p_{1} \in S 1} d\left(p_{1}, S 2\right), \\
& d(S 2, S 1)=\max _{p_{2} \in S 2} d\left(p_{2}, S 1\right),
\end{aligned}
$$

and

$$
\begin{gathered}
d\left(p_{1}, S 2\right)=\min _{p_{2} \in S 2}\left\|p_{1}-p_{2}\right\|, \\
d\left(p_{2}, S 1\right)=\min _{p_{1} \in S 1}\left\|p_{2}-p_{1}\right\| .
\end{gathered}
$$

Note that $\|$. \| denotes the usual Euclidean norm. An illustration of the Hausdorff distance is given in Figure 1, following (ArizaLópez et al., 2011).

\subsection{The octree structure}

The octree is a three-dimensional data structure that recursively subdivides a point cloud within a cubic volume into eight congruent disjoint cubes (octants). An overview of the several types of octrees is given in (Samet, 1988). The subdivision continues until reaching the predetermined level of decomposition.

Given a 3-D point cloud in a metric space, our methodology will rely on: a starting point, $P_{0}$, an ending point, $P_{N}$, that respectively correspond to the closest and farthest point to the origin of the metric space, a subdivision level, and the distance $d\left(P_{N}\right)$ between the starting and ending points. $d\left(P_{N}\right)$ is defined as the maximum between $d_{x}\left(P_{N}\right), d_{y}\left(P_{N}\right)$ and $d_{z}\left(P_{N}\right)$ which are the differences of coordinates between $P_{0}$ and $P_{N}$ along the x-axis, $\mathrm{y}$-axis and z-axis, respectively.

We will now consider a single subdivision level for simplicity. The subdivision algorithm proceeds as follows: initially, the original dataset is sorted by increasing $\mathrm{x}$-axis coordinates and then two subsets are created with respect to a threshold value, which is defined as the sum of the abscissa of the starting point and $d\left(P_{N}\right)$. The first subset will contain all points of the initial cloud

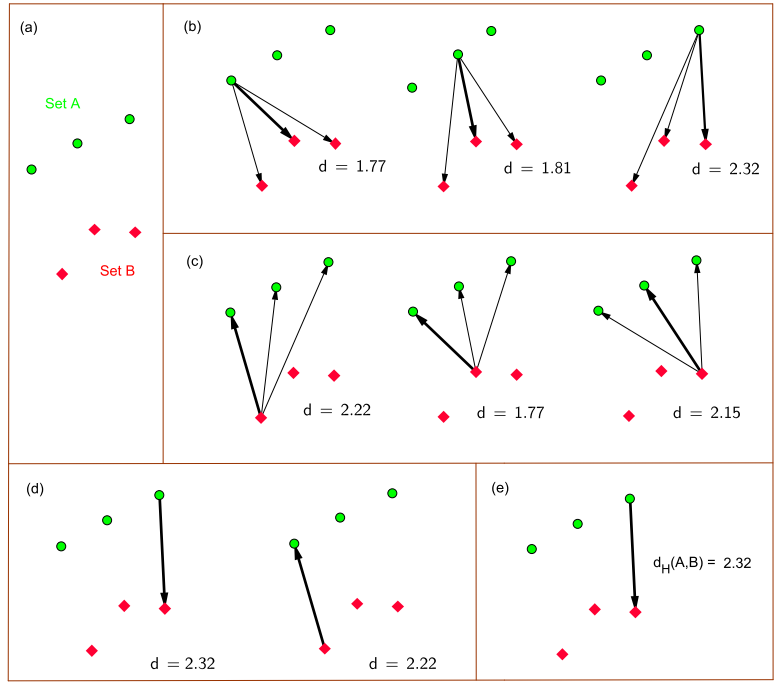

Figure 1: Hausdorff distance for two point sets $A$ and $B$. (a) The point sets. (b) Computation of the Euclidean distance from each element of the set A to each point of B (values in white refer to the thicker arrow corresponding to the minimum distance). (c) Computation of the Euclidean distance from each element of the set B to each point of A (values in white refer to the thicker arrow corresponding to the minimum distance). (d) Maximum distances between sets. (e) Hausdorff distance between set $\mathrm{A}$ and B: $d_{H}(A, B)=2.32$.

with $\mathrm{x}$-coordinate lower than the threshold value and the second one gathers all points with $\mathrm{x}$-coordinates greater than the threshold. Then, the same work is repeated, taking these two subsets as initial data, by sorting them by increasing $y$-axis coordinates and thresholding, which yields four sets of points. Finally these four clouds are sorted again, by increasing $\mathrm{z}$-axis coordinates and a new division takes place according to the already defined characteristics, and one obtains eight octants (see Figure 2 for example).

\subsection{Proposed approaches}

The proposed approaches aim at measuring the accuracy of a 3D model. In this way, we have implemented three algorithms for computing a distance between a set of points and a reference dataset.

Point-to-point algorithm The point-to-point approach aims at comparing two sets of points regularly distributed along the tunnel. The distances are computed only between similar points and the maximum distance is considered. Note that the number of points in both sets must be the same.

Cloud-to-mesh algorithm In the cloud-to-mesh technic, Euclidean distances are computed between points in the photogrammetric model and meshes. The triangular meshes are computed from the lasergrammetric point cloud by Delaunay triangulation. As a consequence, accuracy corresponds to the quadratic mean of all Euclidean distances. It may be noticed that this method presents the risk of being time consuming.

Cloud-to-cloud algorithm In the cloud-to-cloud method, all points of each model (photogrammetric and lasergrammetric) are compared. As we study very large clouds, the computation time between two sets of points may be very high. Thus, we use a specific octree structure (described in Section 3.2). This structure 

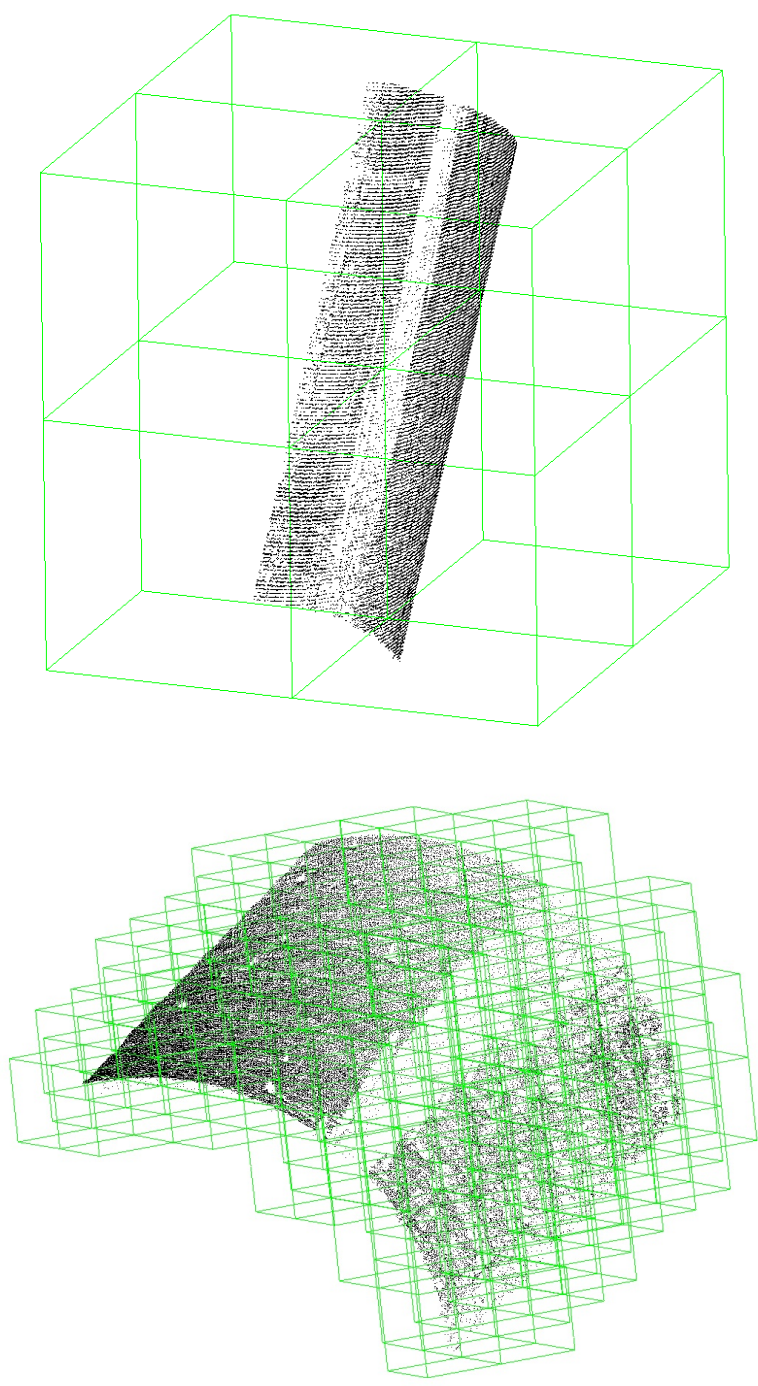

Figure 2: One-level subdivision octree (top) and four-level subdivision (bottom) representation of a portion of the tunnel canal's vault.

is very convenient because it gives the capacity to quickly and accurately address points in a specific cube.

An attractive idea is to compute the Hausdorff distance (detailed in Sec. 3.1) between the octree cells. The accuracy is obtained by calculating the quadratic mean of all the Hausdorff distances. However, the Hausdorff distance is sensitive to the difference of point densities between the two clouds. Moreover, in such a way, the accuracy is estimated using few distances (corresponding to the number of octants) at risk of not being representative of real accuracy.

The alternative method we have developed consists in modeling surfaces using meshing of lasergrammetric cells and then, in introducing locally Euclidean distances between photogrammetric points and laser meshes. It may be specified that a filter is applied to the Euclidean distances in two ways: (1) A minimum of 3 points in a cell is necessary to compute the Euclidean distances. Otherwise the Hausdorff distances is measured between cells ; (2) the Euclidean distances greater than $d_{H}$ are replaced by Hausdorff distances in order to avoid irregularities introduced by triangular meshes. Figure 3 illustrates this algorithm. Accuracy is obtained by calculating the quadratic mean of all the distances.
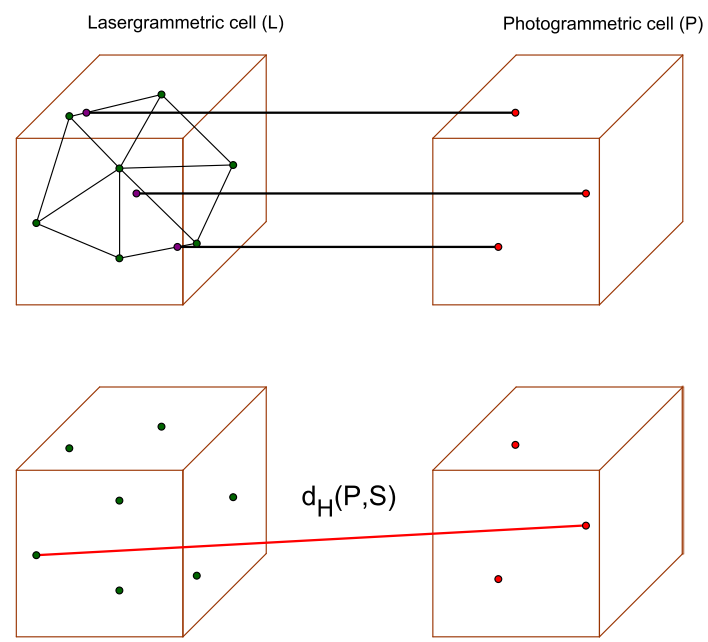

Figure 3: Homologous octree cell representation. Euclidean distances are computed between points of the two clouds (top). Hausdorff distance (bottom) is also computed for filtering the Euclidean distances if required
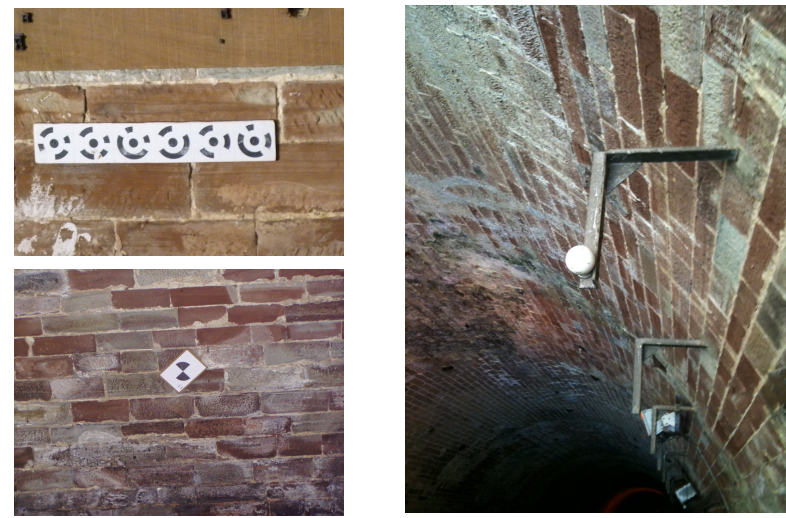

Figure 4: Encoded target for the photogrammetric model (topleft) ; black and white target (bottom-left) ; sphere (right). Encoded targets and spheres are located on the right side wall and black and white targets are fixed on the left side wall.
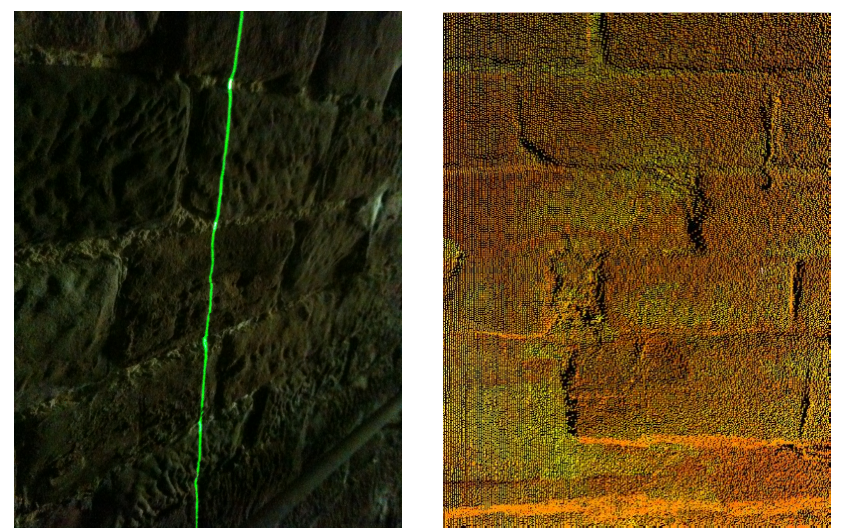

Figure 5: Example of laserscanning of a portion of side wall (left) and resulting point cloud (right). 


\section{DATA RECORDING}

The experimental canal tunnel is located in Niderviller (Lorraine region, France). It is straight, $475 \mathrm{~m}$ long and lined with stonework. To assess the feasibility of non-intrusive acquisition systems, experimental campaigns have been carried out in the tunnel for recording data, both in a dynamic manner for images acquisition and in a static manner for terrestrial laser acquisition. Note that, in parallel to images acquisition, others sensors (tacheometers and inertial unit) have been used to determine the trajectory of the barge in the tunnel, see (Albert et al., 2013). Beforehand, the tunnel has been equipped with 8 spheres, 25 encoded targets and 16 black and white targets (see fig. 4). Moreover, mobile targets were positioned on the ledge of the tunnel during acquisitions. This set of reference points essentially located on the side walls of the tunnel, has been accurately georeferenced using tacheometers. They will be used as control points for georeferencing the photogrammetric and lasergrammetric models.

\subsection{LASERSCANNING}

The static laser acquisition has been performed using a 3D Terrestrial Laser Scanner (a LEICA C10 device) with a station every 30 meters along the ledge of the tunnel. The scanning approach is based on a time-of-flight technology. The field-of-view is $360^{\circ}$ by $270^{\circ}$ and the scanning resolution is 1 point every $5 \mathrm{~mm}$ at a distance of $12 \mathrm{~m}$. A scan records 90 millions of points at every station and lasts 40 minutes (see Fig. 5). We adopt, at least, a $30 \%$ overlap between two adjacent scans in order to facilitate scan strips registration (Bornaz et al., 2003). 15 stations are necessary to collect the laser data of the entire tunnel (1.5 billion points).

Data post-processing consists in building a global terrestrial reference model of the tunnel from different point clouds. In this work, we have chosen a two-step approach. In a first phase, adjacent point clouds are registered using black and white targets, which are visible in two consecutive scans. Secondly, the resulting registered cloud is georeferenced using control points defined by mobile targets. A cleaning-up of the final cloud is necessary to eliminate aberrant points. An exemple point cloud is rendered in Fig 6.

We obtain an extremely dense and non-uniform point cloud, which needs to be resampled. We apply the farthest point sampling strategy (FPS) that locally adapts the sample density according to initial data. We refer the reader to (Eldar et al., 1997, Bronstein et $a l ., 2008)$ for further details on the FPS algorithm.

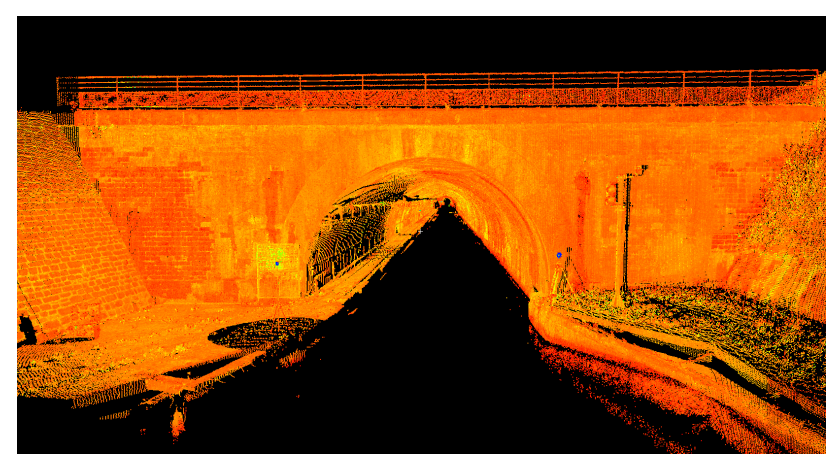

Figure 6: Rendering of the lasergrammetric model at the tunnel exit. No resampling has been applied in this view.

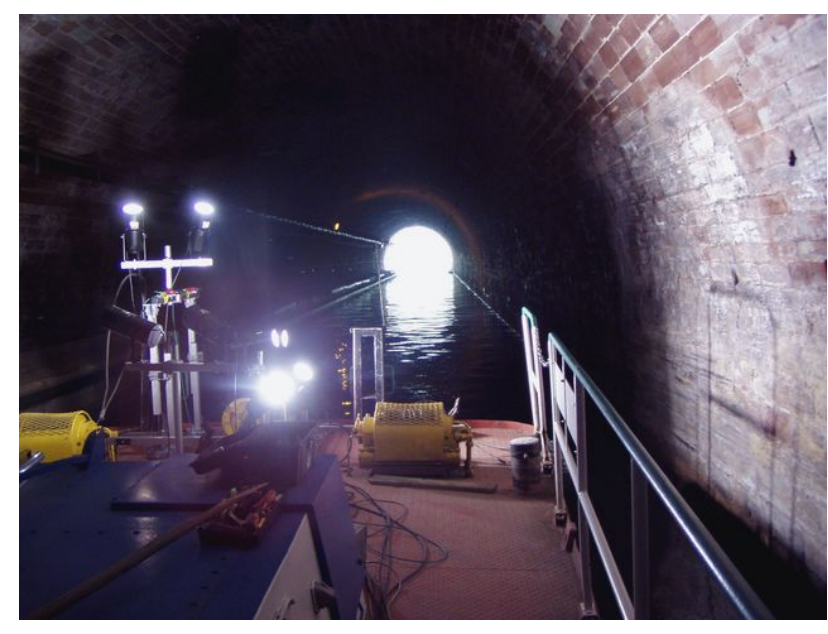

Figure 7: Modular imaging prototype onboard a barge in the experimental tunnel.

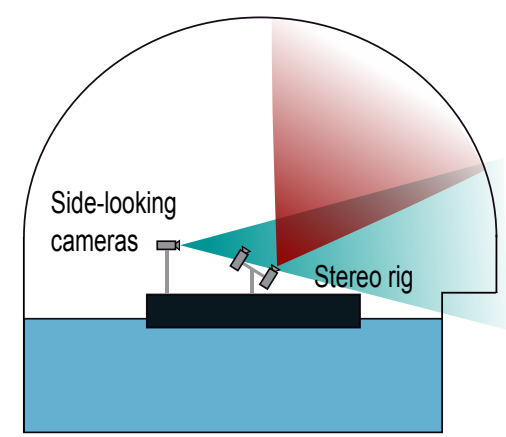

Figure 8: Camera configuration used for modeling the tunnel by photogrammetry.

\subsection{PHOTOGRAMMETRIC MODEL}

Image sequences of the vaults and side walls were dynamically acquired by two pairs of color cameras mounted on a modular structure, onboard a barge. An artificial lighting system has been integrated on the structure to obtain sufficiently illuminated images (see Fig 7). The images recording prototype is detailed in (Albert et al., 2013), in the same Archive.

A sequence is defined as the set of all successive image acquisitions of vaults and side walls along the tunnel. Note that, at every time, only views of the right cameras are used to build the model. There is a small overlap between images of the two systems and an overlap of $75 \%$ between two successive images of the same camera, as shown in figure 8.

The reconstruction of a photogrammetric model of the tunnel is based on the bundle adjustment algorithm. This approach aims at computing an optimal model of a scene from multiple views, by minimizing a quadratic error between actual positions of points in an image and predicted positions obtained by back-projection. Note that this classical technique is a non-linear optimization problem that jointly minimizes the errors in all views (Szeliski, 2010). The algorithm is applied to a set of corresponding points that can be identified in several images. These points are called smartpoints. An example of smartpoints in different views is shown on Fig 9. A second step of the reconstruction consists in scaling the model by using control points, such as encoded 

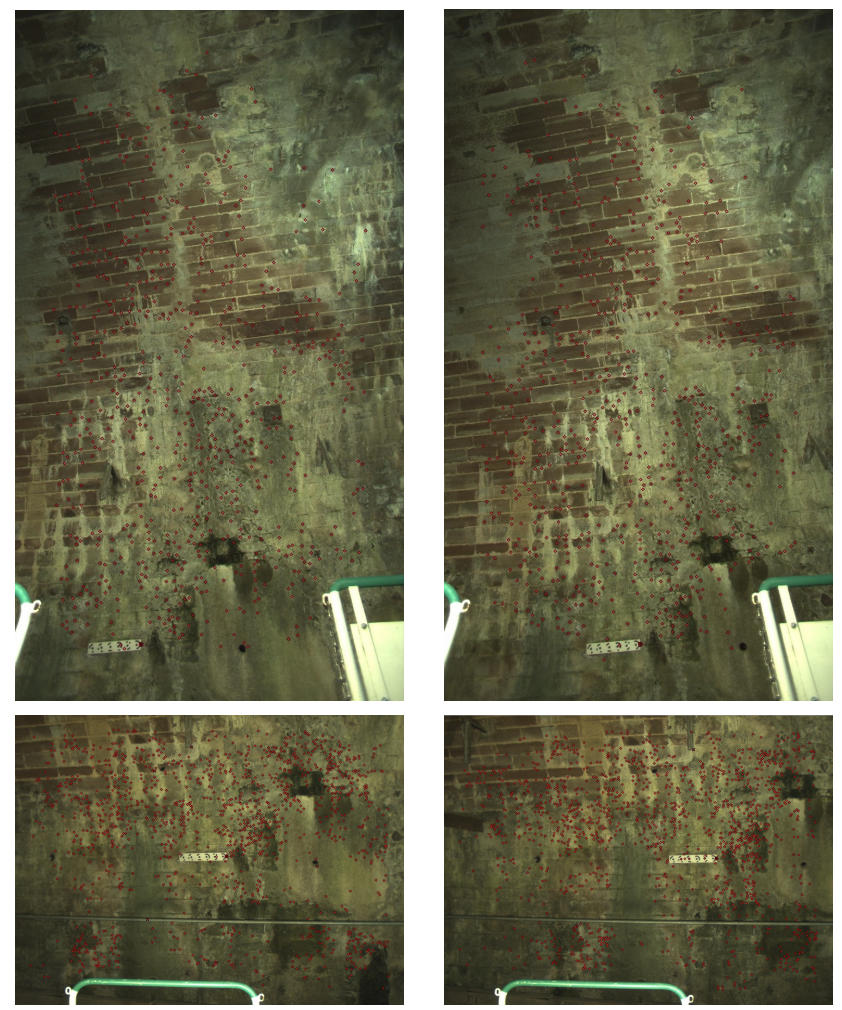

Figure 9: Successive views of the vaults (top) and side wall (bottom) at time $t$ (left) and $t+1$ (right). Smartpoints that have been automatically detected for bundle adjustment are superimposed upon the images (in red).

targets or visual marks, that have been previously georeferenced. These control points are also employed to enforce the model to be straight, to match the actual geometry of the tunnel: without these points, the model tends to be curved, due to local error accumulations. The photogrammetric 3D model of the tunnel was created with the commercial software PhotoModelerScanner $(P M S)$. Actually, as we work on large datasets (500 images by camera), nine sub-models had to be calculated and gathered to obtain a sufficiently dense photogrammetric point cloud of the right side wall and half vault of the entire tunnel.

\section{RESULTS AND DISCUSSION}

The accuracy assessment has been performed on five datasets. The number of points for each dataset is given in Tab. 1. The first two datasets have been built to evaluate accuracy using the pointto-point approach. Dataset 1 is employed to check the accuracy of the TLS point cloud by comparing it to georeferenced points. The six points corresponds to the Leica Geosystems spherical targets, surveyed both by TLS and tacheometry, and regularly spaced along the tunnel. The results give a maximum difference equal to $1.7 \mathrm{~cm}$ that ensures the reliability and the good dispersion of the lasergrammetric model. Therefore, the TLS model can be considered as a reference.

Dataset 2 is created by taking 2 points on each of 51 metallic angle brackets scattered all along the tunnel. The comparison refers to the photogrammetric and TLS model. We determine an accuracy as the maximum distance between the two models. The obtained value is $4 \mathrm{~cm}$, which seems very promising.

The last three datasets have been constituted to evaluate accuracy

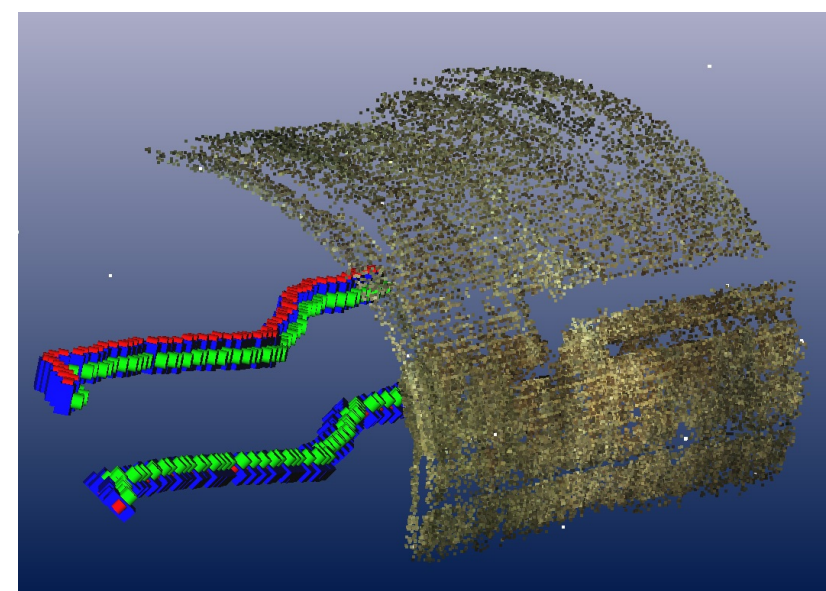

Figure 10: Portion of the 3D model of the side wall and the beginning of the vault obtained with PMS using the sequences of right images (from the side-looking stereo rig and the top-looking stereo rig with $-32^{\circ}$ angle). The poses are shown for each camera location, behind the 3D sparse, textured model.

Table 1: Number of points in both models for each dataset.

\begin{tabular}{|c|c|c|c|}
\hline Datasets & PMS & TLS & Length \\
\hline 1 & & 6 & scattered in tunnel \\
\hline 2 & 102 & 102 & scattered in tunnel \\
\hline 3 & 12507 & 68429 & $18 \mathrm{~m}$ \\
\hline 4 & 63295 & 76578 & $107 \mathrm{~m}$ \\
\hline 5 & 226679 & 201838 & whole tunnel \\
\hline
\end{tabular}

between pairs of point clouds with large and different sizes, using cloud-to-mesh or cloud-to-cloud method. Datasets 3 and 4 are two successive portions of the tunnel starting from its entrance. Note that in dataset 5 , the lasergrammetric point cloud has been resampled as explained in Sect. 4.1. This last dataset, describing the whole 475 meters of the tunnel, presents high disparities in cloud density. It may be noticed that these three datasets correspond to the rightmost half-vault of the tunnel as the photogrammetric model has only been implemented on this part.

The results of the cloud-to-mesh approach are shown in Tab. 2 . The 3 accuracies, comprised in the range $[4.1 \mathrm{~cm}, 5.8 \mathrm{~cm}]$, are homogenous and analogous to the accuracy observed for dataset 2. However, it may be observed that dataset 5 gives an accuracy which is fairly lower than for the two others. This difference can be explained by the resampling and the fact that the whole tunnel was considered.

In the cloud-to-cloud approach, two parameters have to be evaluated: the octree subdivision level and the accuracy measurement. In theory, the choice of the octree subdivision has no impact on the distance computation between clouds. Actually, we observe some accuracy variations according to the subdivision level, but the results are globally homogenous (see Tab. 3). Further investi-

Table 2: "cloud-to-triangular mesh" Method: Accuracy comparison between photogrammetric and TLS model

\begin{tabular}{|c|c|c|}
\hline Datasets & Computation time & Accuracy \\
\hline 3 & $\leq 1 \mathrm{mn}$ & $5.7 \mathrm{~cm}$ \\
\hline 4 & $3.7 \mathrm{mn}$ & $5.8 \mathrm{~cm}$ \\
\hline 5 & $24 \mathrm{mn}$ & $4.1 \mathrm{~cm}$ \\
\hline
\end{tabular}


Table 3: "cloud-to-cloud" method. Accuracy comparison between photogrammetric and TLS model vs. number of octree levels, using dataset 3 .

\begin{tabular}{|c|c|c|}
\hline Octree level & Computation time & Accuracy \\
\hline 3 & $56.47 \mathrm{~s}$ & $5.5 \mathrm{~cm}$ \\
\hline 5 & $20,62 \mathrm{~s}$ & $5.0 \mathrm{~cm}$ \\
\hline 7 & $3.37 \mathrm{~s}$ & $4.1 \mathrm{~cm}$ \\
\hline
\end{tabular}

Table 4: "cloud-to-cloud" method. Accuracy comparison between photogrammetric and TLS model for the three datasets. Seven octree levels are used in this experiment.

\begin{tabular}{|c|c|c|}
\hline Datasets & Computation time & Accuracy \\
\hline 3 & $3.37 \mathrm{~s}$ & $4.1 \mathrm{~cm}$ \\
\hline 4 & $22.32 \mathrm{~s}$ & $5.6 \mathrm{~cm}$ \\
\hline 5 & $8.6 \mathrm{mn}$ & $4.2 \mathrm{~cm}$ \\
\hline
\end{tabular}

gations should be necessary to explain these differences. For the moment, we have chosen, a number of 7 octree levels, since it is the least time consuming.

The evaluation on the datasets $3-5$, taking the laser model as reference, (Tab. 4) shows that the accuracy of the photogrammetric model spans the range $4.1 \mathrm{~cm}-5.5 \mathrm{~cm}$. We observe that the tunnel can be quite accurately reconstructed with a photogrammetric model. It may be observed that, for equivalent results, the computation time using cloud-to-cloud method is lower than using cloud-to-mesh approach. For example, in the case of dataset 5 , the accuracy analysis lasts 24 minutes with cloud-to-mesh algorithm whereas it consumes 8.6 minutes with the octree structure (7 levels) in the cloud-to-cloud method. These results are promising in terms of reliability of photogrammetry approach when images have been dynamically acquired in a long canal-tunnel.

\section{CONCLUSIONS AND FUTURE WORK}

This paper has introduced several methods for point cloud comparison in 3D in the case of canal tunnels. Given two datasets (assuming the clouds are expressed in the same reference frame) acquired either by photogrammetric or lasergrammetric devices, an accurate comparison relying on distance is possible. Speed constraints are tackled using a specific octree subdivision and an appropriate resampling of the point clouds. The first method (point-to-point comparison) allows us to check the quality and reliability of the TLS model. Then, this model can be considered as reference and cloud-to-cloud or cloud-to-triangular mesh techniques can be applied to huge datasets. The results show that the accuracy of the photogrammetry model is around $5 \mathrm{~cm}$ compared to lasergrammetric model considered as the reference.

Algorithms have been designed to deal with TLS and PM data but any sources of point cloud data are possible. They were applied to Niderviller canal tunnel to assess potential changes in photogrammetric survey. Results show that PM model fulfills requirements for reconstructing the tunnel. The choice of appropriate octree structure has to be determined, for instance, by performing simulations. Other subdivision structures, such as $k-d$ trees should be also tested. We are now also working on automatic accuracy assessment for other camera configurations (e.g. panoramic configuration). In the near future, the result of this study will be the creation of a full photogrammetric acquisition device able to evolve in underground waterways.

\section{ACKNOWLEDGEMENTS}

The authors would like to thank Voies Navigables de France and the Centre d'Etudes des Tunnels for funding this project. Many thanks, also, to the students of INSA de Strasbourg who contributed to the project.

\section{REFERENCES}

Albert, J.-L., Charbonnier, P., Foucher, P., Muzet, V., Prybyla, D., Perrin, T., Grussenmeyer, P., Guillemin, S. and Koehl, M., 2013. Devising a visual inspection system for canal tunnels: Preliminary studies. In: XXIV International CIPA Symposium, Strasbourg, France.

Ariza-López, F., Mozas-Calvache, A., Ureña-Cámara, M., AlbaFernández, V., García-Balboa, J., Rodríguez-Avi, J. and RuizLendínez, J., 2011. Influence of sample size on line-based positional assessment methods for road data. ISPRS Journal of Photogrammetry and Remote Sensing 66(5), pp. 708-719.

Aspert, N., Santa-Cruz, D. and Ebrahimi, T., 2002. Mesh: Measuring errors between surfaces using the hausdorff distance. In: Proceedings ofIEEE International Conference on Multimedia and Exposition, Vol. 1, IEEE, Lausanne, Switzerland, pp. 705-708.

Bornaz, L., Lingua, A. and Rinaudo, F., 2003. Multiple scan registration in lidar close range applications. Int. Arch. Photogram. Rem. Sens. Spatial Inform. Sci 34, pp. 72-77.

Bronstein, A. M., Bronstein, M. M. and Kimmel, R., 2008. Numerical geometry of non-rigid shapes. Springer, ISBN: 978-0$387-73300-5$

Cignoni, P., Rocchini, C. and Scopigno, R., 1998. Metro: measuring error on simplified surfaces. Computer Graphics Forum 17(2), pp. 167-174.

Eldar, Y., Lindenbaum, M., Porat, M., Member, S. and Zeevi, Y. Y., 1997. The farthest point strategy for progressive image sampling. IEEE Trans. on Image Processing pp. 1305-1315.

Girardeau-Montaut, D., Roux, M., Marc, R. and Thibault, G., 2005. Change detection on points cloud data acquired with a ground laser scanner. International Archives of Photogrammetry, Remote Sensing and Spatial Information Sciences 36(part 3), pp. 30-35.

Han, J.-Y. and Jiang, J. G. Y.-S., 2013. Monitoring tunnel profile by means of multi-epoch dispersed 3-d lidar point clouds. Tunnelling and Underground Space Technology 33, pp. 186-192.

Kim, K.-Y., Kim, C.-Y., Lee, S.-D., Seo, Y. and Lee, C., 2007. Measurement of tunnel 3-d displacement using digital photogrammetry. J Korean Soc Eng Geol 17(4), pp. 567-576.

Lague, D., Brodu, N. and Leroux, J., 2013. Accurate 3D comparison of complex topography with terrestrial laser scanner: application to the rangitikei canyon (nz). ISPRS Journal of Photogrammetry remote sensing 82 , pp. 10-26.

Mémoli, F. and Sapiro, G., 2004. Comparing point clouds. In: Proceedings of the 2004 Eurographics/ACM SIGGRAPH symposium on Geometry processing, ACM, pp. 32-40.

Pejić, M., 2013. Design and optimisation of laser scanning for tunnels geometry inspection. Tunnelling and Underground Space Technology 37, pp. 199-206.

Samet, H., 1988. An overview of quadtrees, octrees, and related hierarchical data structures. In: Theoretical Foundations of Computer Graphics and CAD, Springer, pp. 51-68.

Szeliski, R., 2010. Computer Vision: Algorithms and Applications. 1st edn, Springer-Verlag New York, Inc., New York, NY, USA. 